\title{
Modeling Instrumental Field-Dependent Aberrations in the NIRC2 Instrument on the Keck II Telescope
}

\author{
Breann N. Sitarski ${ }^{a}$, Gunther Witzel ${ }^{a}$, Michael P. Fitzgerald ${ }^{a}$, Leo Meyer ${ }^{a}$, Andrea M. Ghez ${ }^{a}$, \\ Randall D. Campbell ${ }^{b}$, Jessica R. Lu ${ }^{c}$, Keith Matthews ${ }^{d}$, Peter Wizinowich ${ }^{b}$, Jim Lyke ${ }^{b}$ \\ ${ }^{a}$ Department of Physics and Astronomy, University of California, Los Angeles, 430 Portola \\ Plaza, Los Angeles, CA 90095-1547, USA; \\ ${ }^{b}$ W. M. Keck Observatory, 65-1120 Mamalahoa Hw., Kamuela, HI 96743, USA; \\ ${ }^{c}$ Institute for Astronomy, University of Hawaii, 2680 Woodlawn Drive, Honolulu, HI 96822, \\ USA; \\ ${ }^{d}$ Division of Physics, Mathematics, and Astronomy, California Institute of Technology, \\ Pasadena, CA 91125, USA
}

\begin{abstract}
We present a model of field-dependent aberrations arising in the NIRC2 instrument on the W. M. Keck II telescope. We use high signal-to-noise phase diversity data employing a source in the Nasmyth focal plane to construct a model of the optical path difference as a function of field position and wavelength. With a differential wavefront error of up to $190 \mathrm{~nm}$, this effect is one of the main sources of astrometric and photometric measurement uncertainties. Our tests of temporal stability show sufficient reliability for our measurements over a 20-month period at the field extrema. Additionally, while chromaticity exists, applying a correction for field-dependent aberrations provides overall improvement compared to the existing aberrations present across the field of view.
\end{abstract}

Keywords: Telescopes: Keck II, Astronomical instrumentation: Cameras-NIRC2, Adaptive Optics: fielddependent aberrations

\section{INTRODUCTION AND GOALS}

Anisoplanatism and the field-dependence of the anisoplanatic point spread function (PSF) greatly influences astrometric and photometric accuracy. In crowded fields like the Galactic Center, insufficient knowledge of the PSF variation accounts for the largest error in astrometric measurements (Witzel et al., this volume). The varying morphologies affect not only astrometric analyses, but photometric analyses as well. As in our first publication on the project, ${ }^{1}$ we divide the definition of the optical transfer function (OTF) into two parts: a static, instrument-based term, and the Adaptive Optics (AO) response to the atmospheric PSF term that takes into account the $C_{n}^{2}$ profile of the atmospheric turbulence.

A static, instrument-based term can still have field dependences and can be further decomposed into on- and off-axis terms. ${ }^{1}$ The on-axis term is expected to be estimated by other means, such as on-sky phase diversity (Jolissant et al., this volume, and Ragland et al., this volume) or through crowded-field estimation. We present a fiber phase diversity phase map grid with data taken between October 2012 and June 2014 that uses an improved data collection methodology. We study the effects of time variability in our instrumental data as well as the affects of chromaticity in our data and discuss whether we can apply this Fe II-based grid to other data sets, including broadband $K^{\prime}$ data.

B. N. S.: E-mail: bsitarski@astro.ucla.edu, Telephone: 1-310-825-3172 


\section{FIBER PHASE DIVERSITY DATA COLLECTION}

We work to measure the differential instrumental aberrations in NIRC2 with phase diversity data taken with a illuminated fiber internal to NIRC2 itself. By measuring the phase of aberrations at a specific position on the detector, we aim to transform phases at specific points to phases at arbitrary field points. Keck Observatory currently uses phase diversity to optimize static aberrations in their image sharpening procedure, which is run prior to every adaptive optics (AO) night at Keck Observatory on the NIRC2 instrument. In order to extract the phase at each point, an image is taken at $\Delta \mathrm{z}=-2 \mathrm{~mm},-4 \mathrm{~mm}$, and $-6 \mathrm{~mm}$, where $\Delta \mathrm{z}$ is difference in defocus term.

Traditionally, image sharpening only needs to focus on low-order modes, and phase diversity collection made use of short exposure times ( $t_{\text {int }}=0.181 \mathrm{sec}, n_{\text {coadds }}=50$, and the CDS readout mode). While this method is efficient in terms of total integration time spent at each field position (72 seconds), it produces a very low signal-to-noise ratio phase diversity map (Figure 1). This is, in large part, due to insufficient integration times for the more out-of-focus images that are used when the phase is extracted.

Aiming to obtain higher signal-to-noise ratio phase maps, we developed an improved method of data collection. Instead of keeping the integration time constant for each $\Delta z$, we increased the integration times and number of reads and decreased the number of coadds (Table 1). While increasing the overall integration time (213 seconds), this yields a phase diversity map with only $12.5 \mathrm{~nm}$ RMS random measurement error in each phase map.

Table 1. An Improved Method for Phase Diversity data collection

\begin{tabular}{llll}
\hline$\Delta \mathrm{z}(\mathrm{mm})$ & $t_{\text {int }}(\mathrm{sec})$ & $n_{\text {coadds }}$ & $n_{\text {reads }}$ \\
\hline 0 & 0.181 & 50 & CDS \\
\hline-2 & 1.5 & 20 & 8 \\
\hline-4 & 7 & 6 & 16 \\
\hline-6 & 180 & 2 & 64 \\
\hline
\end{tabular}

In order to get the phase at each position, we image sharpen at the center (at pixel [545, 495] measured from zero-based index) then take phase diversity data across the field of view. By extracting phase information at various points, we can measure the field dependence of the aberrations.
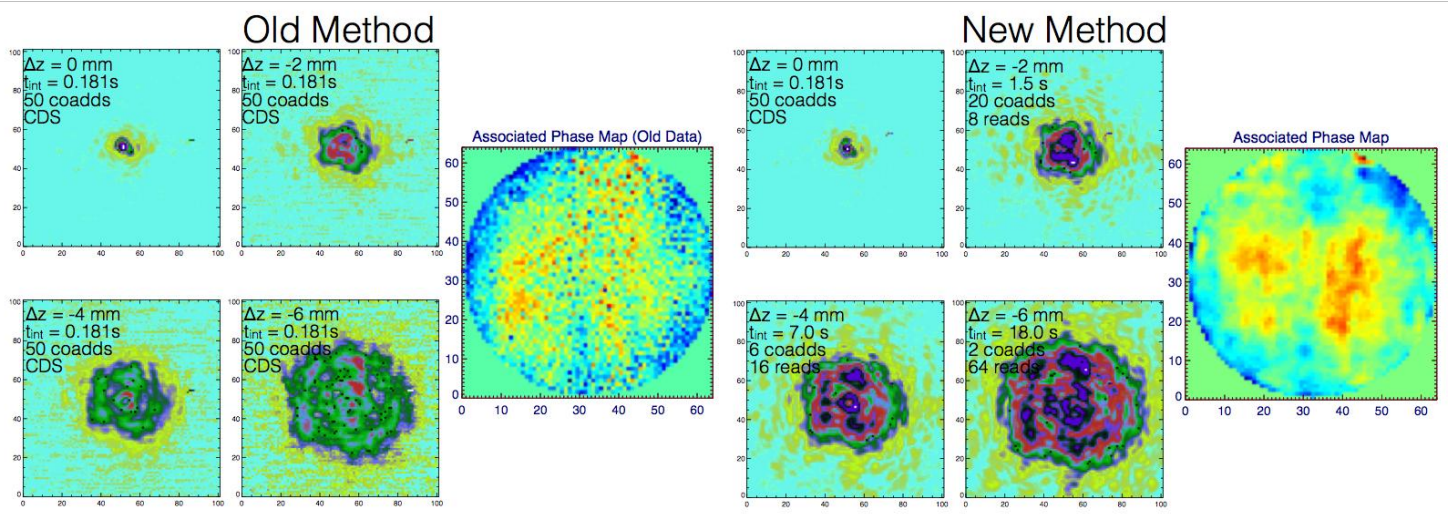

Figure 1. A comparison between the old method of collection Phase Diversity data and our new method, which results in a much higher signal-to-noise ratio phase map.

\subsection{Wavefront Error and Field Variation Parameters}

In total, we have taken $\sim 55$ hours of fiber phase diversity data across the field of view of NIRC2 in the fashion described in the above section. This yields approximately 150 phase diversity phase maps, some taken at the 
same position over various times to measure the affects of time variability on the data (see section $\mathrm{X}$ ). The full grid of phase diversity data is shown in Figure 2, where each phase map is centered at the position it was acquired. All of these data were taken at the Fe II $\left(\lambda_{0}=1.65 \mu \mathrm{m}\right)$ narrow-band filter, following the image sharpening procedure at Keck Observatory. The effects of chromaticity are described in section X.

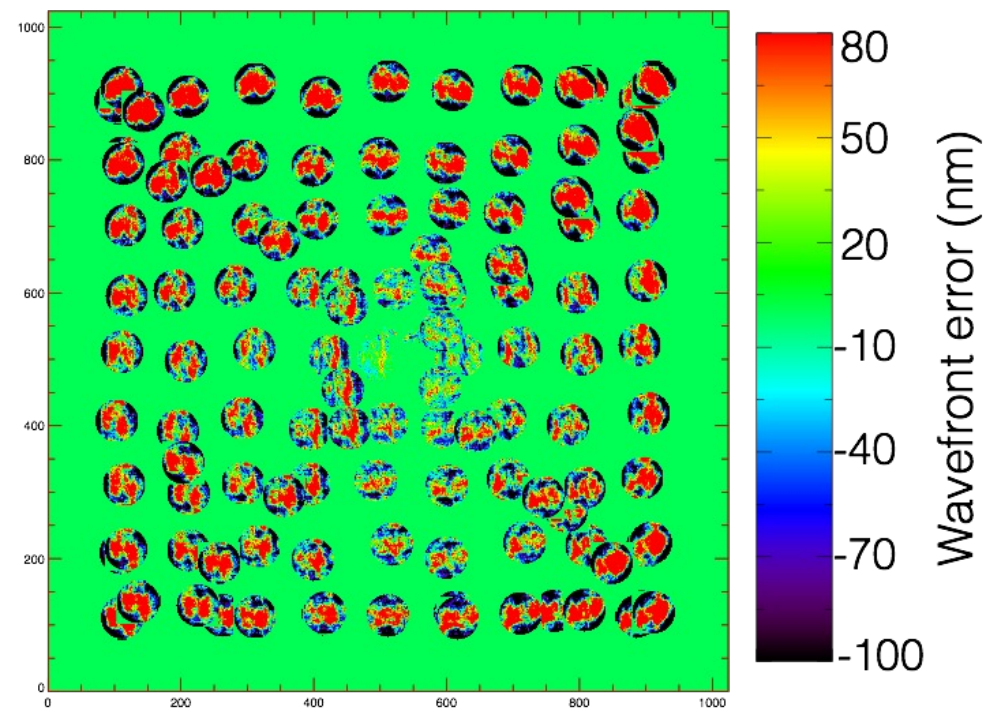

Figure 2. Our full phase diversity data grid, where phase diversity data is taken at various points across the field of view. There are 150 different data sets (some taken at the same position to study time variability affects). All data is taken through the Fe II narrow-band filter on $\operatorname{NIRC} 2\left(\lambda_{0}=1.65 \mu \mathrm{m}\right)$.

At each field position, we take three phase diversity data sets (including the OPD map, and images at $\Delta \mathrm{z}=$ $0,-2,-4$, and $-6 \mathrm{~mm}$ ), allowing us to determine the errors on the Zernike terms. We acquire a center position data set $([\mathrm{x}, \mathrm{y}]=[545,495])$ at the beginning and end of each data acquisition run which are averaged together. The center position is then subtracted out from all other field points for that specific data acquisition run in order to obtain the differential position with regards to the center position.

The field extrema points are those furthest from the center position-the four corners of the detector. The relative wavefront error between the center reference point and the corner points is $190 \mathrm{~nm}$ RMS at Fe II. In fact, the wavefront error difference between adjacent samples can be used to quantify the error associated with the field sampling. The average wavefront error difference is $43 \mathrm{~nm}$ RMS at Fe II. The smallest and largest wavefront error differences between adjacent OPD maps is 87 and $15 \mathrm{~nm}$ RMS at Fe II, respectively.

Adjacent samples allow us to quantify the error associated with field sampling. Figure 3 shows a map and the connecting points of the adjacent phase maps with their wavefront error; Figure 3 also plots the difference in RMS Wavefront Error as a function of the distance to the adjacent phase map. While the maps with nearby neighbors have fairly low error (below the temporal error), they exhibit quite a lot of scatter. This can be caused by field variation because phase maps near extrema may differ more drastically than those close to the image sharpening position. The average wavefront error difference between adjacent samples is $43 \mathrm{~nm}$ RMS, while the smallest/largest WFE difference between adjacent samples is 15 and $87 \mathrm{~nm}$ RMS, respectively. These all fall below the average wavefront error difference relative to the center of the field (96.5 $\mathrm{nm}$ RMS).

\section{WAVELENGTH VARIATION OF PHASE DIVERSITY DATA}

We take all of our phase diversity data at the narrow-band Fe II filter $\left(\lambda_{0}=1.6544 \mu \mathrm{m} ; \Delta \lambda=0.0256 \mu \mathrm{m}\right)$. However, all Galactic Center data is taken through the $K^{\prime}\left(\lambda_{0}=2.124 \mu \mathrm{m}, \Delta \lambda=0.351 \mu \mathrm{m}\right)$ and $L^{\prime}\left(\lambda_{0}=3.776 \mu \mathrm{m}, \Delta \lambda=0.700\right.$ $\mu \mathrm{m})$ filters. Furthermore, all Keck image sharpening data is also taken at Fe II; it is therefore necessary to see if the phase diversity phase map grid outlined in section $\mathrm{X}$ is usable at other wavelengths. 

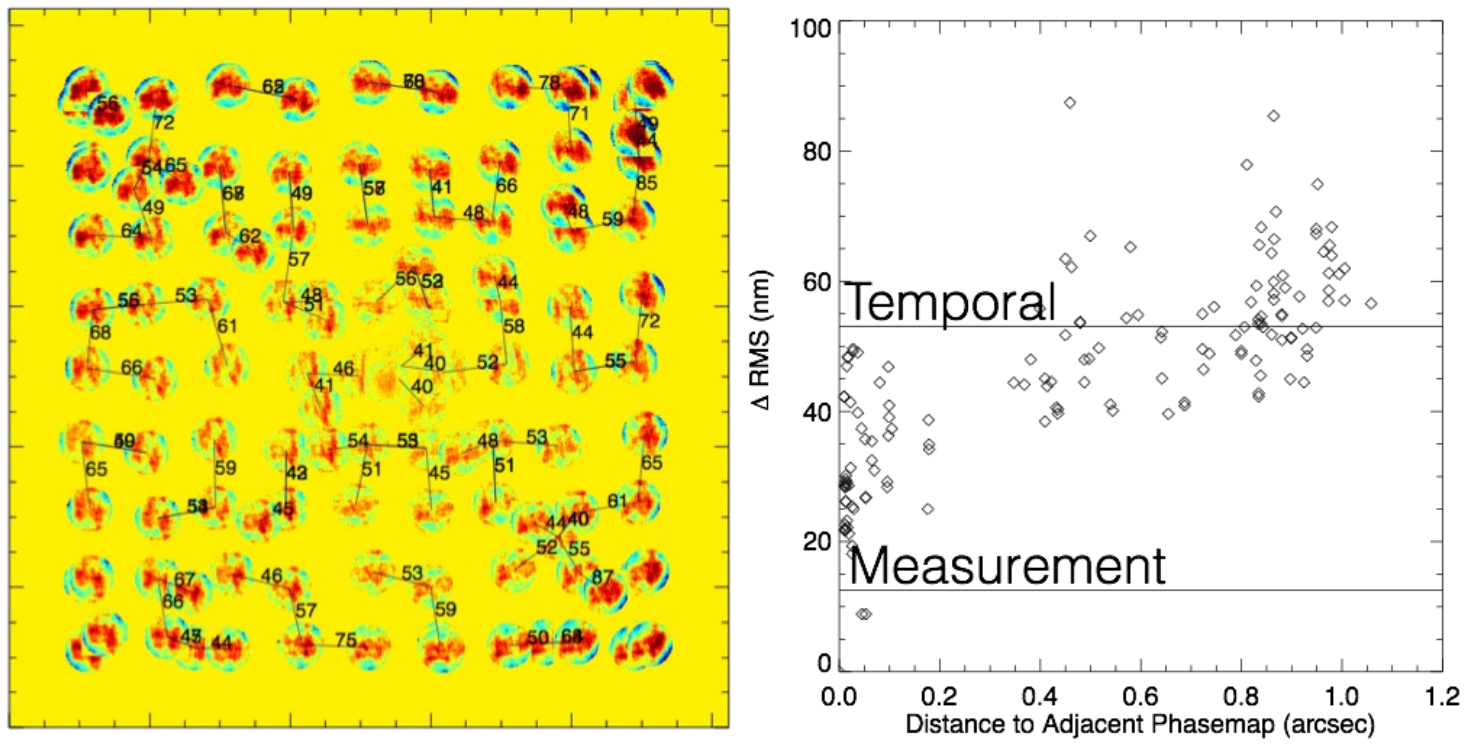

Figure 3. Grid of adjacent phase maps and the associated wavefront error between the adjacent samples.

We took phase diversity data at several non-thermal narrow- and medium-band filters available on NIRC2: He $1 \mathrm{~A}\left(\lambda_{0}=1.0847 \mu \mathrm{m} ; \Delta \lambda=0.0182 \mu \mathrm{m}\right) ; \operatorname{Pa} \gamma\left(\lambda_{0}=1.096 \mu \mathrm{m} ; \Delta \lambda=0.016 \mu \mathrm{m}\right) ; \mathrm{J}$ continuum $\left(\lambda_{0}=1.2132 \mu \mathrm{m}\right.$; $\Delta \lambda=0.0198 \mu \mathrm{m}) ; \mathrm{Pa} \beta\left(\lambda_{0}=1.2903 \mu \mathrm{m} ; \Delta \lambda=0.0193 \mu \mathrm{m}\right) ; \mathrm{H}$ continuum $\left(\lambda_{0}=1.5804 \mu \mathrm{m} ; \Delta \lambda=0.0232 \mu \mathrm{m}\right) ; \mathrm{CH}_{4} \mathrm{~S}$ $\left(\lambda_{0}=1.5923 \mu \mathrm{m} ; \Delta \lambda=0.1257 \mu \mathrm{m}\right) ; \mathrm{Fe}$ II; $\mathrm{CH}_{4} \mathrm{~L}\left(\lambda_{0}=1.6809 \mu \mathrm{m} ; \Delta \lambda=0.1368 \mu \mathrm{m}\right) ;$ He $1 \mathrm{~B}\left(\lambda_{0}=2.0563 \mu \mathrm{m}\right.$; $\Delta \lambda=0.0326 \mu \mathrm{m}) ; \operatorname{Br} \gamma\left(\lambda_{0}=2.1686 \mu \mathrm{m} ; \Delta \lambda=0.0326 \mu \mathrm{m}\right)$; and $\mathrm{K}$ continuum $\left(\lambda_{0}=2.2706 \mu \mathrm{m} ; \Delta \lambda=0.0296 \mu \mathrm{m}\right)$. We acquired the phase diversity using the method described in Section X. However, we optimized integration times to obtain less than $10,000 \mathrm{DN} /$ coadd since nonlinearity becomes an issue with the NIRC2 detector.

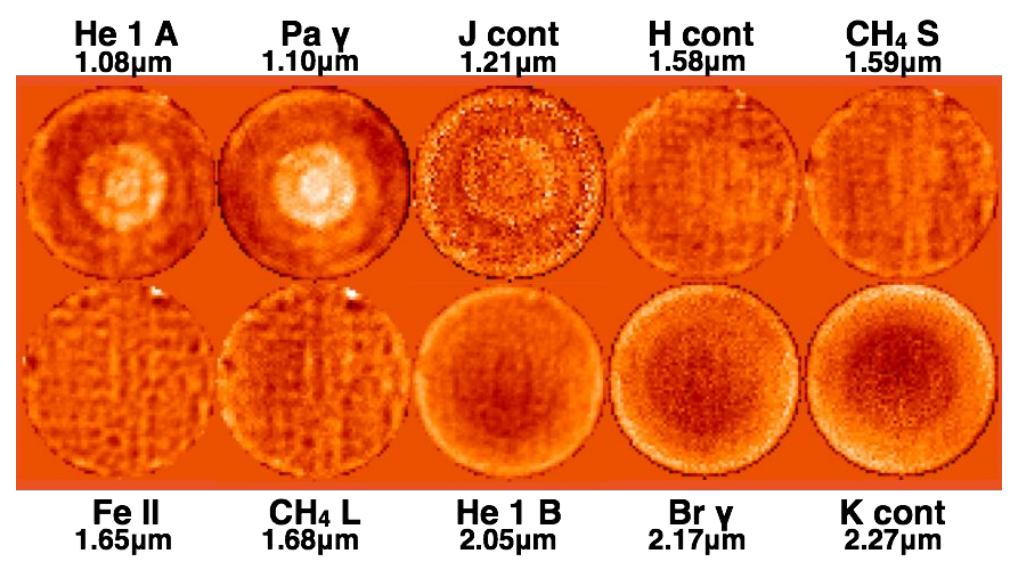

Figure 4. Center phase maps extracted from data taken at various narrow- and medium-band filters available on NIRC2. There is a visible apparent focus offset.

Assuming that the phase maps extracted from the phase diversity data are linear combinations of the Zernike polynomials, we extract the defocus term from each phase map, as it is visually apparent that there is some defocus offset (see Figure 4). In plotting this defocus term as a function of wavelength, some sort of dependence of defocus on wavelength becomes apparent (Figure 5). Keck Observatory has implemented a focus compensation for closed loop operation on sky to account for the focus offset. The defocus could arise due to a transmissive 
slab in a converging beam (e.g., dichroic, window).

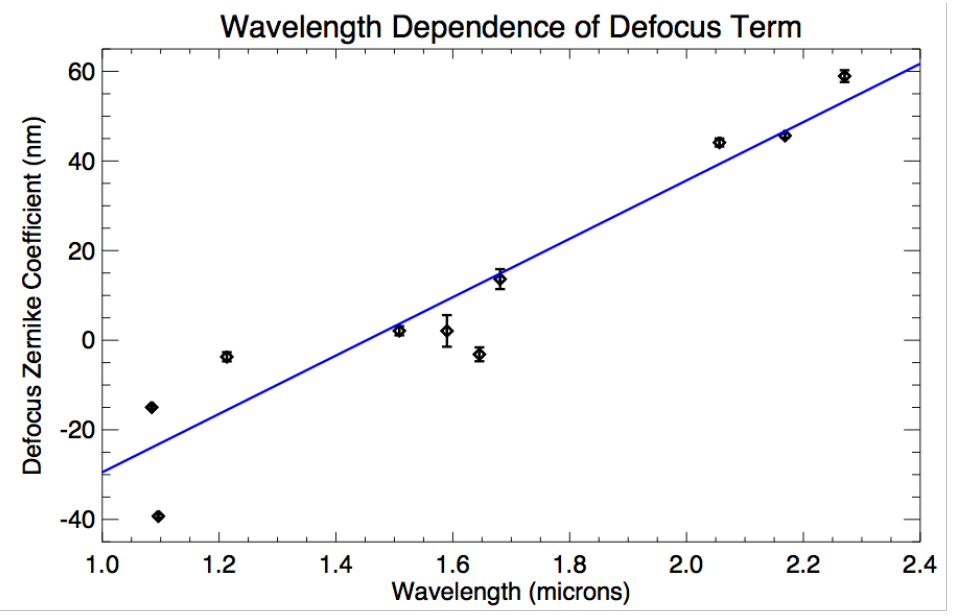

Figure 5. A plot of the extracted Zernike coefficient for the defocus mode as a function of wavelength. There is a linear trend between the wavelength and magnitude of the defocus term.

\section{COMPATIBILITY OF FE II AND BR $\gamma$ FIELD OPD MAPS}

Since our entire grid of phase diversity data is currently taken at the Fe II filter, we need to determine if we can extend the use of our grid to different wavelengths, primarily since the majority of our Galactic Center science data is taken at the broadband $K^{\prime}$ filter $\left(\lambda_{0}=2.124 \mu \mathrm{m} ; \Delta \lambda=0.351 \mu \mathrm{m}\right) .{ }^{2-7}$

Comparing the field extrema (the corner s of the detector) allows us to investigate the wavelength dependence of the aberrations. After subtracting out the respective center phase maps for a given wavelength from the extrema phase maps, we compare the $\mathrm{Br} \gamma$ and Fe II optical path differences. A Zernike decomposition of the optical path differences is shown in Figure 6 . The $\mathrm{Br} \gamma$ and Fe II are comparable to within $\sim 80 \mathrm{~nm}$ RMS. While that is a large value, it overall much less than the $190 \mathrm{~nm}$ extrema-to-center difference. Even so, it is still more advantageous to apply this instrumental model with the Fe II data than to not apply the model at all.

Due to the brightness of the fiber, $K^{\prime}$ data was not obtained at the field extrema since we had to subarray down to a $320 \times 312$ grid around the very center of the NIRC2 detector in order to not saturate the in-focus $\Delta \mathrm{z}=0 \mathrm{~mm}$ image. We obtained positions at the extrema of this subarray, and decomposed them in the way described above but at $K^{\prime}$ and corresponding field positions at Fe II. The Zernike decomposition is shown in Figure 7. The largest optical path differences is $50.9 \mathrm{~nm}$ RMS.

\section{TIME VARIABILITY}

The time difference between acquiring many of the field positions, ranging from October 2012 to June 2014, poses a major concern in using a grid of phase diversity data. In order to probe the effects of time variability, we took multiple data sets at the field extrema and the center positions over this time period.

We took the center position twice over a span of five hours to probe the temporal drift on short time periods. Since we take three sets of data (and therefore three phase maps) at each position, we use an average phase map for each time and subtract them to solve for the residual. The temporal drift over 5 hours is therefore $18 \mathrm{~nm}$ RMS (see Figure 8). Between October 2012 and June 2014, the temporal drift between the center phase maps is $41.6 \mathrm{~nm}$ RMS, obtained using a similar analysis as described above. The measurement repeatability at the center positions is $12.5 \mathrm{~nm}$ RMS.

We carried out a similar exercise at the field extrema. Between October 2012 and May 2014, the temporal drift over the field extrema is $53.1 \mathrm{~nm}$ RMS, with a measurement repeatability of $15.43 \mathrm{~nm}$ RMS. It is noteworthy 


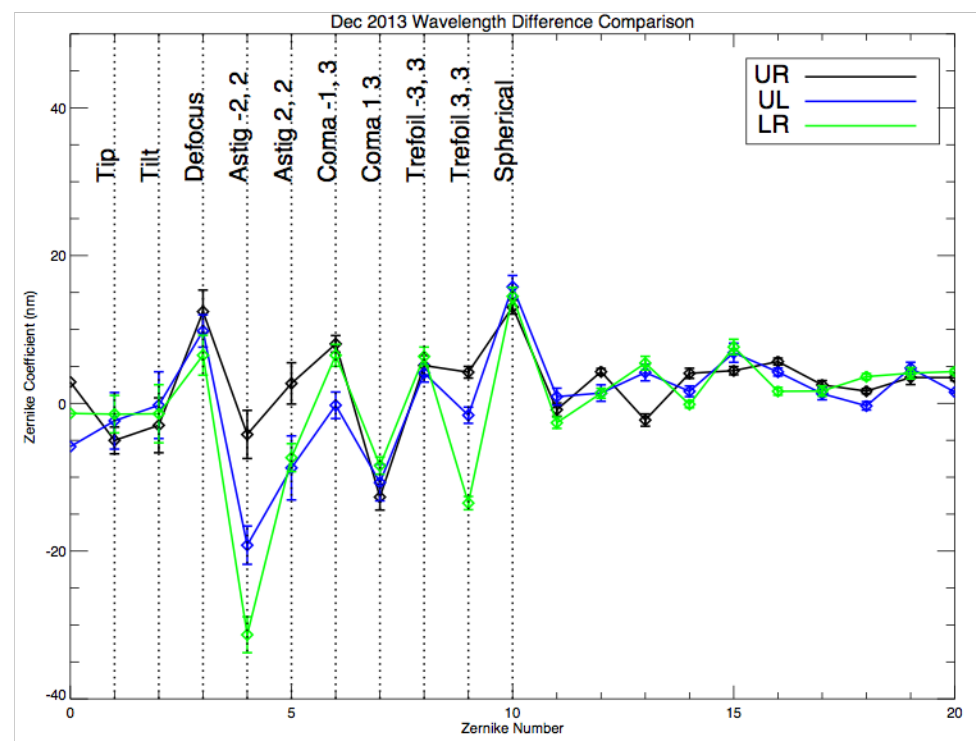

Figure 6. Zernike decomposition of the optical path differences between $\mathrm{Br} \gamma$ and Fe II at three of the field extrema. The lower-order terms clearly dominate the overall wavefront error.

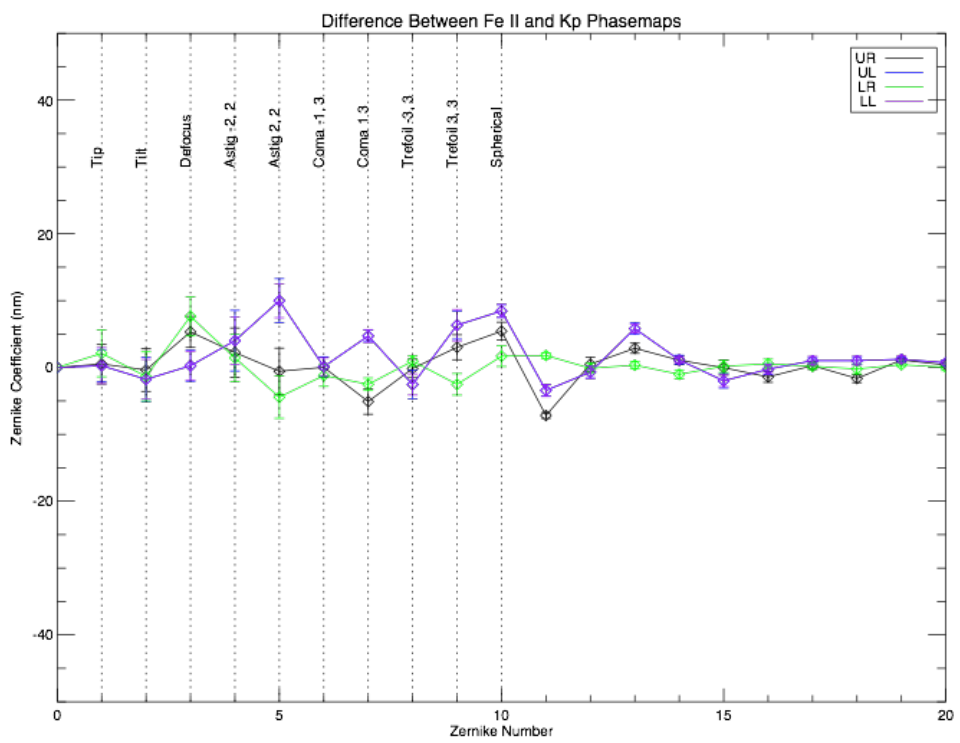

Figure 7. Zernike decomposition of the optical path differences between $K^{\prime}$ and Fe II at positions fairly close to the center of the detector that stayed in the $320 \times 312$ subarray of the detector. The lower-order terms again dominate the overall wavefront error.

that the $53.1 \mathrm{~nm}$ RMS temporal difference falls significantly below the $190 \mathrm{~nm}$ RMS center to corner difference in phase maps (see Figure 9).

However, it appears that much of the error is common mode. The top row of Figure 10 shows phase maps from three different epochs. At each extrema point, we averaged over all the epochs and subtracted that to yield the second row. To determine if there were any common-mode errors in a given epoch, we averaged the results of the second row over a given epoch and subtracted it from row 2. This results in field-dependent wavefront error stability after removing common-mode errors of $<20 \mathrm{~nm}$ RMS. 


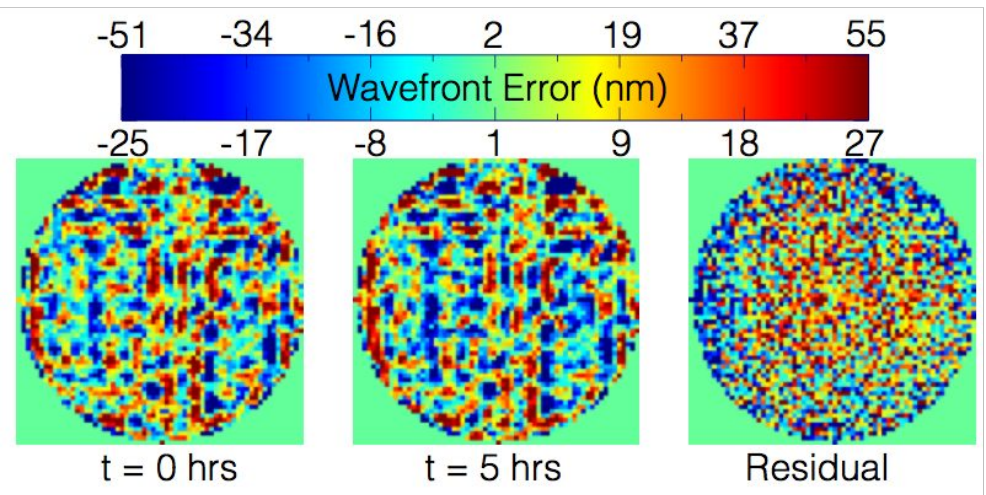

Figure 8. Variation of the center phase map over 5 hours. The residual seems to consist of high-order noise.

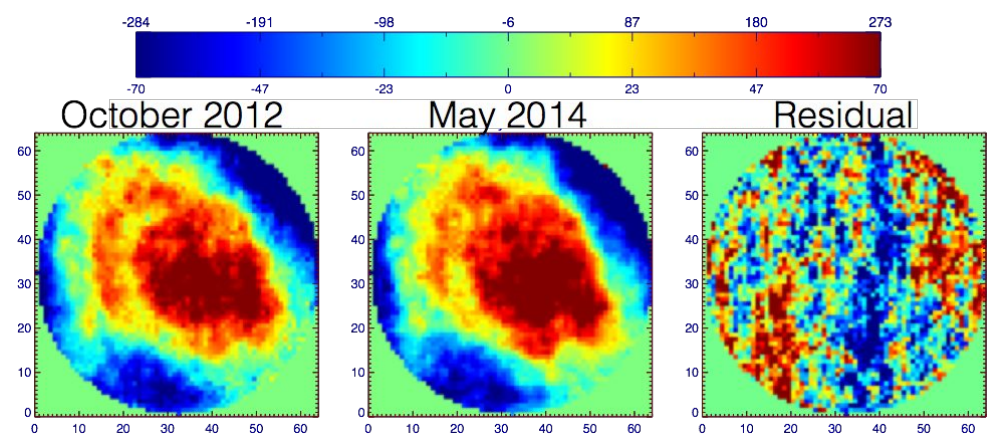

Figure 9. Variation of the one of the extrema points (corner positions) as a function of time. The general morphology of the phase map stays the same, but the residual seems to consist of high-order noise.

\section{ON-SKY TESTS AND VALIDATION}

In Figure 11, we show an on-sky test of the instrumental model that we have developed. The observed on-axis PSF is the guide star for the Natural Guide Star AO system we utilized on the Keck II telescope. ${ }^{8,9}$ We transform the observed on-axis PSF to the model shown on the far right of the image by multiplying by the OTF ratio generated from the nearest phase maps of the on-axis and off-axis points. It is clear from the comparison of the middle frame (observed off-axis) and the far-right frame (model) that many complex features are reproduced in our model, as denoted by the arrows.

\section{CONCLUSIONS AND FUTURE WORK}

We have presented a large phase diversity data collection effort to map the aberrations across the field of view of the NIRC2 detector on the Keck II telescope. This data collection consists of a grid of phase diversity data taken at Fe II $\left(\lambda_{0}=1.65 \mu \mathrm{m}\right)$, along with multi-wavelength studies and temporal studies from October 2012 to June 2014.

Our efforts have shown that while time variability and chromaticity have an effect, their are intrinsically less than simply not correcting for the field-dependent aberrations at all arising from the instrumental PSF (see Table 2). In fact, as shown in Figure 5, we may be able to calibrate out the lower-order modes causing a discrepancy between $\mathrm{Br} \gamma$ and Fe II by using a method similar for calibrating out the defocus term.

Future work includes expanding this work to create an interpolated model for each Zernike coefficient across the field of view of the NIRC2 detector. Preliminary results are favorable to this method and would allow for correction not be based on the nearest phase diversity phase map as we are currently implementing. We will combine this work with models of the AO response to the atmospheric PSF for both Laser Guide Star AO and Natural Guide Star AO and apply it to both crowded regions and other science cases (Lu et al., this volume). 


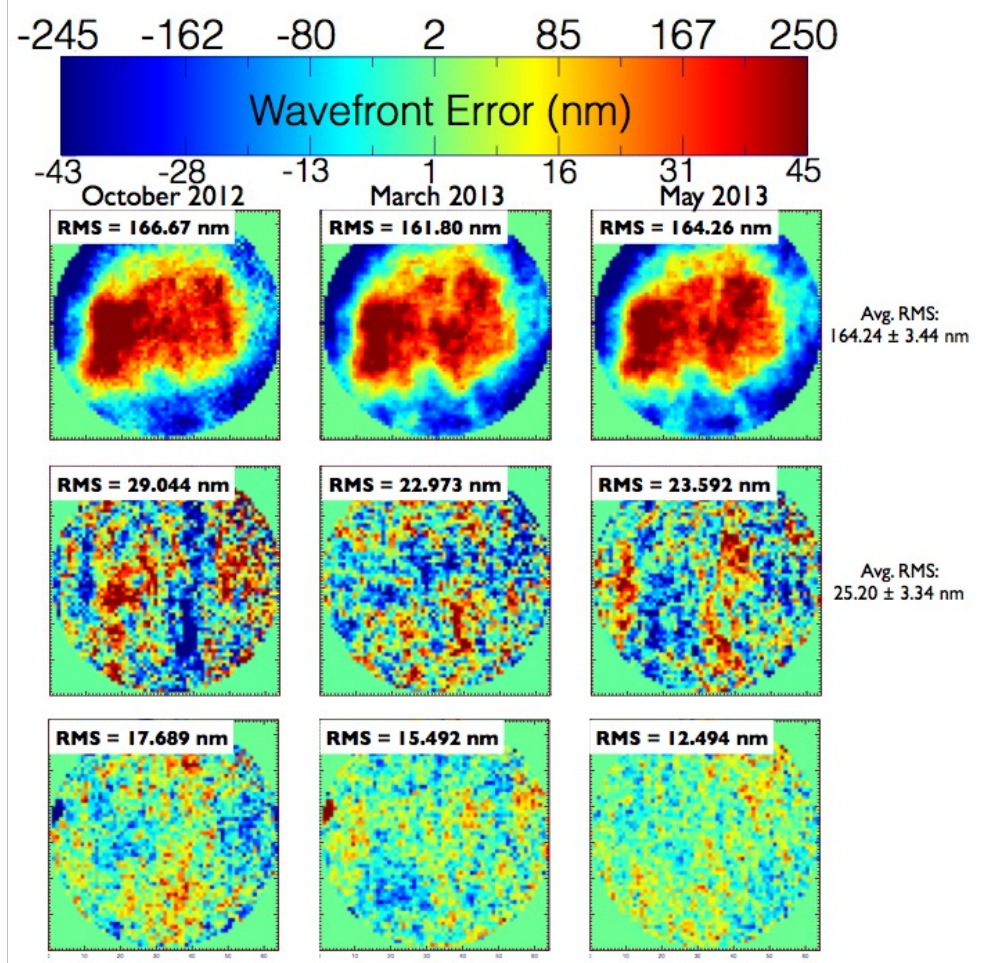

Figure 10. There seems to be common-mode error that exists across the different epochs and different field points. The middle row shows the average of the top row subtracted out; the last row shows the average of all extrema data at the respective epoch subtracted out.
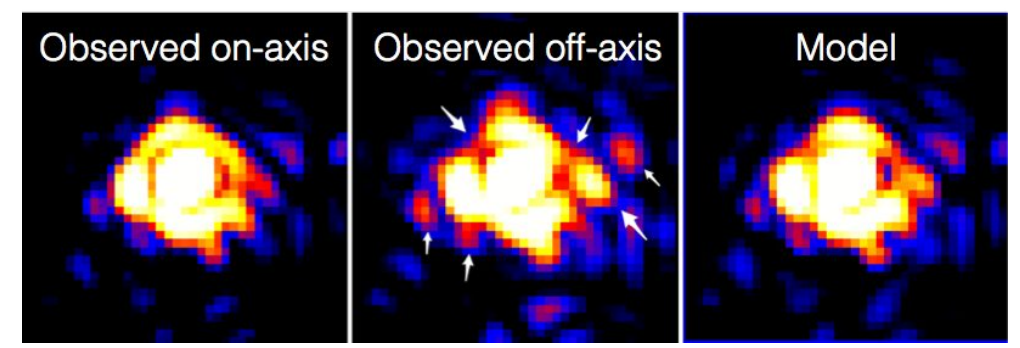

Figure 11. Transformation of the on-axis PSF to the model. Complex structure from the observed off-axis PSF is reproduced by our model in this binary star test.

\section{ACKNOWLEDGEMENTS}

We would like to acknowledge the financial support of the W. M. Keck Foundation for this project. AMG and MM are also supported by NSF AST-0909218. The authors would also like to thank Jay Anderson, Richard Dekaney, Brent Ellerbroek, James Graham, Claire Max, Andrei Tokovinin, and Marcos van Dam for their valuable input to the project. BNS would also like to thank Saundra Albers.

\section{REFERENCES}

[1] Fitzgerald, M. P., Witzel, G., Britton, M. C., Ghez, A. M., Meyer, L., Sitarski, B. N., Cheng, C., Becklin, E. E., Campbell, R. D., Do, T., Lu, J. R., Matthews, K., Morris, M. R., Neyman, C. R., Tyler, G. A., Wizinowich, P. L., and Yelda, S., "Modeling anisoplanatism in the Keck II laser guide star AO system," Proc. SPIE 8447 (July 2012). 
Table 2. An Improved Method for Phase Diversity data collection

\begin{tabular}{ll}
\hline Measurement & Wavefront Error (WFE; nm RMS) \\
\hline Center to Extrema max WFE Difference & 190 \\
\hline Temporal drift of center phase map over 5 hours & 18 \\
\hline Temporal drift of center from October 2012 - June 2014 & 41.6 \\
\hline Temporal drift of field extrema from October 2012 - May 2014 & 53.1 \\
\hline Measurement repeatability of center phase map & 12.5 \\
\hline Measurement repeatability of field extrema phase maps & 15.4 \\
\hline Compatibility of Br $\gamma$ and Fe II at corners & 80 \\
\hline Compatibility of $K^{\prime}$ and Fe II at $K^{\prime}$ corners & 50.9 \\
\hline Average WFE Difference between adjacent maps & 43 \\
\hline Smallest/largest WFE difference between adjacent maps & $15 / 87$ \\
\hline Average WFE difference relative to the center over the field & 96.5 \\
\hline
\end{tabular}

[2] Ghez, A. M., Hornstein, S. D., Lu, J. R., Bouchez, A., Le Mignant, D., van Dam, M. A., Wizinowich, P., Matthews, K., Morris, M., Becklin, E. E., Campbell, R. D., Chin, J. C. Y., Hartman, S. K., Johansson, E. M., Lafon, R. E., Stomski, P. J., and Summers, D. M., "The First Laser Guide Star Adaptive Optics Observations of the Galactic Center: Sgr A*'s Infrared Color and the Extended Red Emission in its Vicinity," 635, 10871094 (Dec. 2005).

[3] Ghez, A. M., Salim, S., Weinberg, N. N., Lu, J. R., Do, T., Dunn, J. K., Matthews, K., Morris, M. R., Yelda, S., Becklin, E. E., Kremenek, T., Milosavljevic, M., and Naiman, J., "Measuring Distance and Properties of the Milky Way's Central Supermassive Black Hole with Stellar Orbits," 689, 1044-1062 (Dec. 2008).

[4] Lu, J. R., Ghez, A. M., Hornstein, S. D., Morris, M. R., Becklin, E. E., and Matthews, K., "A Disk of Young Stars at the Galactic Center as Determined by Individual Stellar Orbits," 690, 1463-1487 (Jan. 2009).

[5] Yelda, S., Ghez, A. M., Lu, J. R., Do, T., Meyer, L., and Morris, M. R., "Adaptive optics observations of the galactic center young stars," Proc. SPIE 8447 (July 2012).

[6] Yelda, S., Ghez, A. M., Lu, J. R., Do, T., Meyer, L., Morris, M. R., and Matthews, K., "Properties of the Remnant Clockwise Disk of Young Stars in the Galactic Center," 783, 131 (Mar. 2014).

[7] Lu, J. R., Do, T., Ghez, A. M., Morris, M. R., Yelda, S., and Matthews, K., "Stellar Populations in the Central 0.5 pc of the Galaxy. II. The Initial Mass Function," 764, 155 (Feb. 2013).

[8] Wizinowich, P. L., Le Mignant, D., Bouchez, A. H., Campbell, R. D., Chin, J. C. Y., Contos, A. R., van Dam, M. A., Hartman, S. K., Johansson, E. M., Lafon, R. E., Lewis, H., Stomski, P. J., Summers, D. M., Brown, C. G., Danforth, P. M., Max, C. E., and Pennington, D. M., "The W. M. Keck Observatory Laser Guide Star Adaptive Optics System: Overview," 118, 297-309 (Feb. 2006).

[9] van Dam, M. A., Bouchez, A. H., Le Mignant, D., Johansson, E. M., Wizinowich, P. L., Campbell, R. D., Chin, J. C. Y., Hartman, S. K., Lafon, R. E., Stomski, Jr., P. J., and Summers, D. M., "The W. M. Keck Observatory Laser Guide Star Adaptive Optics System: Performance Characterization," 118, 310-318 (Feb. 2006). 